\title{
Valued outranking relations in ELECTRE providing manageable disaggregation procedures
}

\author{
Vincent Mousseau ${ }^{\mathrm{a}, \mathrm{b}, *}$, Luis Dias ${ }^{\mathrm{c}, \mathrm{d}, 1}$ \\ a DIMACS, Rutgers University, CoRE Building, 96 Frelinghuysen Road, Piscataway, NJ 08854-8018, USA \\ b LAMSADE, Université Paris Dauphine, Place du Maréchal De Lattre de Tassigny, 75775 Paris Cedex 16, France, \\ ${ }^{c}$ Faculty of Economics, University of Coimbra, Av. Dias da Silva, Coimbra, Portugal \\ ${ }^{\mathrm{d}}$ INESC-Coimbra, R. Antero de Quental 199, Coimbra, Portugal
}

Received 10 December 2001; accepted 21 January 2003

\begin{abstract}
In ELECTRE methods, the construction of an outranking relation amounts at validating or invalidating, for any pair of alternatives $(a, b)$, the assertion " $a$ is at least as good as $b$ ". This comparison is grounded on the evaluation vectors of both alternatives, and on additional information concerning the decision maker's preferences, accounting for two conditions: concordance and non-discordance.

In decision processes using these methods, the analyst should interact with the decision maker in order to elicit values for preferential parameters. This can be done either directly or through a disaggregation procedure that infers the parameters values from holistic judgements provided by the decision maker. Inference is usually performed through an optimization program that accounts for the aggregation model and minimizes an "error function". Although disaggregation approaches have been largely used in additive models, only few advances have been made towards a disaggregation approach for outranking methods. Indeed, outranking methods may lead to computationally difficult inference problems.

In this paper we are concerned with a slight adaptation of the valued outranking relation used in the ELECTRE III and ELECTRE TRI. Such modification is shown to preserve the original discordance concept. We show that the modified outranking relation makes it easier to solve inference programs.
\end{abstract}

(c) 2003 Elsevier B.V. All rights reserved.

Keywords: Multiple criteria analysis; Valued outranking relations; Veto effect; Parameter inference procedures; ELECTRE

\section{Introduction}

In the field of multiple criteria decision aiding (MCDA), a class of methods ground the recommendations to the decision maker (DM) on the construction of one (or several) binary relation(s) representing the preference among pairs of alternatives (see [24,25]) rather than on the construction of a synthesizing utility

\footnotetext{
${ }^{*}$ Corresponding author. Tel.: +33-144-05-44-01; fax: +33-144-05-40-91.

E-mail addresses: mousseau@lamsade.dauphine.fr (V. Mousseau), ldias@pombo.inescc.pt (L. Dias).

${ }^{1}$ Tel.: 351-239-790500.
} 
function (see [10]). These methods are usually referred as outranking methods in the MCDA literature and belong to the so-called European school of MCDA (see [26]). Let us consider a decision situation involving a finite set of alternatives $A=\left\{a_{1}, a_{2}, \ldots, a_{l}\right\}$ evaluated on $n$ criteria $g_{1}, g_{2}, \ldots, g_{n}(F=\{1,2, \ldots, n\}$ denotes the set of criteria indices; we will assume without loss of generality that preferences are increasing with the value on $g_{j}$, i.e., the greater $g_{j}(a)$ the better $a$ ).

The construction of an outranking relation $S$ amounts at validating or invalidating, for any pair of alternatives $(a, b) \in A^{2}$, an assertion $a S b$, whose meaning is " $a$ is at least as good as $b$ " or synonymously " $a$ is not worse than $b$ ". This comparison is grounded on the evaluation vectors of both alternatives $a$ and $b$, i.e., $\left(g_{1}(a), g_{2}(a), \ldots, g_{n}(a)\right)$ and $\left(g_{1}(b), g_{2}(b), \ldots, g_{n}(b)\right)$, and on additional information concerning the DM's preferences. To validate a statement $a S b$, two basic conditions should be verified: concordance and non-discordance (or non-veto).

A criterion $g_{k}$ is said to be concordant with the assertion $a S b$ if $a$ is at least as good as $b$ with respect to criterion $g_{k}$. The concordance condition is fulfilled for the assertion $a S b$ iff the subset of criteria concordant with $a S b$ is "sufficiently" large. A criterion $g_{k}$ is said to oppose a veto to the assertion $a S b$ if the difference of evaluation $g_{k}(b)-g_{k}(a)$ is incompatible with the assertion $a S b$, whatever the evaluation on the other criteria. The non-discordance condition is fulfilled iff no criterion opposes a veto to the assertion $a S b$.

Several outranking methods using these concepts of concordance and non-discordance (see [21]) have been proposed in the literature (see $[2,12,19,20,24,27]$ ) and put these concepts into a concrete form in different ways.

In a decision process using these methods, the analyst usually interacts with the DM in order to elicit values for preference-related parameters. This can be done either directly or through a disaggregation procedure that infers the parameters values from holistic preferences provided by the DM (see [9]). Inference is usually performed through an optimization program that accounts for the aggregation model and minimizes an "error function". This disaggregation approach has been largely used in additive models (e.g. see [8]). However, only few advances have been made towards a disaggregation approach for outranking methods (see $[11,13,17]$ ). A possible reason for this is that the outranking methods, namely the well-known ELECTRE methods [25], originated from real-world applications and are rather "optimization unfriendly", i.e., lead to parameter inference programs that are frequently non-linear and difficult due to non-convexity.

Various alternative implementations of the concordance/non-discordance ideas have been proposed ([21] defines a wide class of operators). In this paper, we are concerned with some adaptations of the valued outranking relation used in ELECTRE III (see [23]) and ELECTRE TRI (see $[18,25,28])$ that preserves the original ideas and is more optimization-friendly for parameter inference programs. More specifically, the modification proposed concerns the implementation of the non-discordance concept. The aim of this modification is to reduce the computational difficulty of the mathematical program to be solved so as to infer the parameters values from holistic preferences. The modifications are also useful for robustness analysis [5] and other approaches [13]. The paper is organized as follows. The first section will describe how the ELECTRE III and ELECTRE TRI methods define the outranking relation $S$ emphasizing the nature of the difficulties for disaggregation procedures. Modifications of ELECTRE's outranking relation in the way it accounts for veto phenomena are proposed in Section 4. Such modifications are shown to preserve the original discordance concept. Section 5 shows that the modified outranking relations make it easier to solve inference programs, namely for the ELECTRE TRI method.

\section{The valued outranking relation in ELECTRE}

\subsection{Outranking relations for a single criterion}

ELECTRE builds a valued outranking relation $S_{j}$ restricted to a single criterion for each criterion $g_{j}$. $S_{j}(a, b)$ is defined by (1) on the basis of $g_{j}(a), g_{j}(b)$ and two thresholds functions: indifference $q_{j}\left(g_{j}\right)$ and 


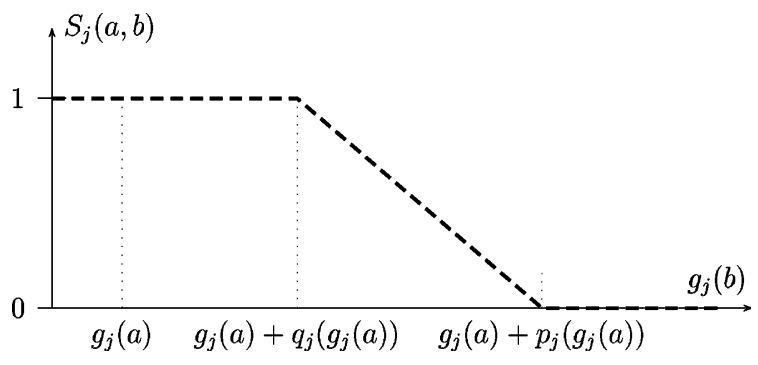

Fig. 1. Partial valued outranking relation.

preference $p_{j}\left(g_{j}\right)\left(0<q_{j}\left(g_{j}\right)<p_{j}\left(g_{j}\right)\right) .{ }^{2} S_{j}(a, b)$ represents the degree to which alternative $a$ outranks (is at least as good as) $b$ (see Fig. 1).

$$
S_{j}(a, b)=\frac{p_{j}\left(g_{j}(a)\right)-\min \left\{g_{j}(b)-g_{j}(a), p_{j}\left(g_{j}(a)\right)\right\}}{p_{j}\left(g_{j}(a)\right)-\min \left\{g_{j}(b)-g_{j}(a), q_{j}\left(g_{j}(a)\right)\right\}} .
$$

\subsection{Concordance relation}

The valued concordance relation $C(a, b)$ is grounded on the relations $S_{j}(j=1,2, \ldots, n)$ and represents the level of majority among the criteria in favor of the assertion " $a$ is at least as good as $b$ ". When computing this majority level, each criterion $g_{j}$ has a weight $w_{j} \geqslant 0$ representing its voting power. Without any loss of generality, we will consider $\sum_{j=1}^{n} w_{j}=1$. Therefore, $C(a, b)$ can be written as follows:

$$
C(a, b)=\sum_{j=1}^{n} w_{j} S_{j}(a, b) .
$$

\subsection{Discordance relation for a single criterion}

ELECTRE builds a valued discordance relation $d_{j}$ restricted to a single criterion for each criterion $g_{j}$. Each $d_{j}(a, b)$ is defined by (3) on the basis of $g_{j}(a), g_{j}(b)$, a veto threshold function $v_{j}\left(g_{j}\right)$ and the preference threshold function $p_{j}\left(g_{j}\right)\left(p_{j}\left(g_{j}\right)<v_{j}\left(g_{j}\right)\right) .{ }^{3}$ (see Fig. 2).

$$
d_{j}(a, b)=1-\frac{v_{j}\left(g_{j}(a)\right)-\min \left\{g_{j}(b)-g_{j}(a), v_{j}\left(g_{j}(a)\right)\right\}}{v_{j}\left(g_{j}(a)\right)-\min \left\{g_{j}(b)-g_{j}(a), p_{j}\left(g_{j}(a)\right)\right\}} .
$$

\subsection{Overall non-discordance relation}

The valued discordance relation $\mathrm{ND}(a, b)$ is grounded on $C(a, b)$ and on the relations $d_{j}, j=1,2, \ldots, n$, it represents the degree to which the minority criteria (i.e., criteria that express a preference in favor of $b$ over $a$ ) collectively oppose a veto to the assertion " $a$ is at least as good as $b$ ". A classical way of defining $\mathrm{ND}(a, b)$ (see [24]) is given in (4). $\mathrm{ND}(a, b)=0$ corresponds to a situation where the minority criteria are totally opposed to $a S b$ whereas $\operatorname{ND}(a, b)=1$ means that none of the criteria oppose a veto to $a S b$.

\footnotetext{
${ }^{2}$ We will consider $q_{j}\left(g_{j}\right)<p_{j}\left(g_{j}\right)$, although ELECTRE also considers the case $q_{j}\left(g_{j}\right)=p_{j}\left(g_{j}\right)$.

${ }^{3}$ We will consider $p_{j}\left(g_{j}\right)<v_{i}\left(g_{j}\right)$, although ELECTRE also considers the case $p_{j}\left(g_{j}\right)=v_{j}\left(g_{j}\right)$.
} 


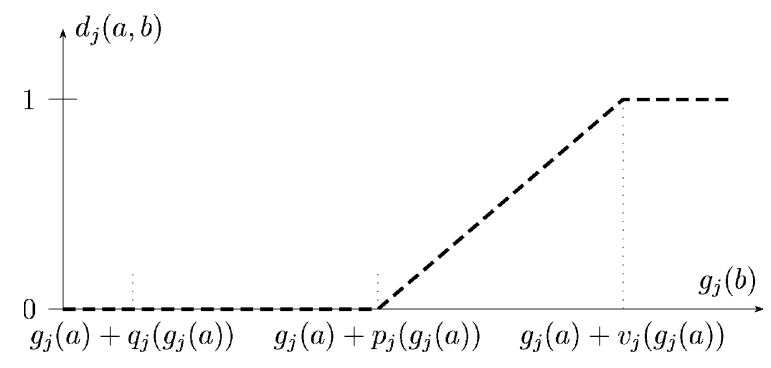

Fig. 2. Partial valued outranking relation.

$$
\mathrm{ND}(a, b)=\prod_{j \in \bar{F}} \frac{1-d_{j}(a, b)}{1-C(a, b)} \quad \text { where } \quad \bar{F}=\left\{j \in F \mid d_{j}(a, b)>C(a, b)\right\} .
$$

We give hereafter in (5)-(7) an equivalent formula to define $\operatorname{ND}(a, b)$. Such definition will be helpful in order to compare $S(a, b)$ with the new outranking relation $S^{\prime}(a, b)$ we define in Section 3. Let us define $\mathrm{ND}_{j}(a, b), j \in F, a, b \in A$, as

$$
\mathrm{ND}_{j}(a, b)=\left\{\begin{array}{lll}
1 & \text { if } \quad d_{j}(a, b) \leqslant C(a, b) \\
\frac{1-d_{j}(a, b)}{1-C(a, b)} & \text { if } \quad d_{j}(a, b)>C(a, b) .
\end{array}\right.
$$

Lemma. Definition (5) is equivalent to

$$
\operatorname{ND}_{j}(a, b)=\operatorname{Min}\left\{1, \frac{1-d_{j}(a, b)}{1-C(a, b)}\right\}
$$

Proof. When $d_{j}(a, b)>C(a, b)$, then it holds $\left(1-d_{j}(a, b)\right) /(1-C(a, b))<1$, thus $\operatorname{ND}_{j}(a, b)=$ $\left(1-d_{j}(a, b)\right) /(1-C(a, b))$; when $d_{j}(a, b) \leqslant C(a, b)$, then it holds $\left(1-d_{j}(a, b)\right) /(1-C(a, b)) \geqslant 1$, thus $\mathrm{ND}_{j}(a, b)=1$. Let us remark that we can state $C(a, b)<1$, as the case $C(a, b)=1$ corresponds to a situation where no discordant criterion exists.

We can then formulate $\mathrm{ND}(a, b)$ as in (7) (equivalent to (4)) in which the product considers each criterion in $F$.

$$
\mathrm{ND}(a, b)=\prod_{j \in F} \mathrm{ND}_{j}(a, b)
$$

\subsection{Valued outranking relation}

ELECTRE combines the concordance and non-discordance relations in order to define the outranking relation $S$ as shown in (8):

$$
S(a, b)=C(a, b) \mathrm{ND}(a, b) .
$$


From the valued outranking relation $S(a, b)$, it is possible to define a family of nested crisp outranking relations $S_{\lambda}\left(S_{\lambda}=\{(a, b) \in A \times A: S(a, b) \geqslant \lambda\}, \lambda \in[0.5,1]\right)$; these crisp relations correspond to $\lambda$-cuts of $S(a, b)$, where the cutting level $\lambda$ represents the minimum value for $S(a, b)$ so that $a S_{\lambda} b$ true (see [7] for more details).

\subsection{Discussion on the ELECTRE valued outranking relation}

\subsubsection{Implementation of the non-discordance principle}

Let us analyze the way the non-discordance condition is implemented through $\mathrm{ND}(a, b)$. If $g_{j}(b)-g_{j}(a)$ exceeds $v_{j}\left(g_{j}(a)\right)$ for at least one criterion then $a S b$ is invalidated, i.e., $\exists j \in F: d_{j}(a, b)=1 \Rightarrow S(a, b)=0$. Furthermore, the partial discordance indices $d_{j}(a, b)$ are defined in such a way that veto effects (i.e., situations in which $d_{j}(a, b)>0$ ) can occur even when $g_{j}(b)-g_{j}(a)<v_{j}\left(g_{j}(a)\right)$. However, to avoid accounting for low values of $d_{j}(a, b)$, the overall non-discordance relation defined in (4) considers the $d_{j}(a, b)$ only for criteria such that $d_{j}(a, b)>C(a, b)$.

Another specific feature of $\operatorname{ND}(a, b)$ lies in the fact that its value accounts both for the values of $d_{j}(a, b)$ and $C(a, b)$ : the way $\mathrm{ND}(a, b)$ accounts for $d_{j}(a, b)$ is amplified when $C(a, b)$ is low. The reason for this is that a veto situation should be accentuated when the concordance relation is not firmly established.

\subsubsection{Difficulties with integrating $S(a, b)$ in inference programs}

In order to elicit values for preference-related parameters (i.e., $w_{j}, v_{j}\left(g_{j}\right), p_{j}\left(g_{j}\right), q_{j}\left(g_{j}\right)$, and limits of categories in ELECTRE TRI) it is possible to proceed using a disaggregation procedure that infers the parameters values from holistic preferences provided by the DM. Hence, it is necessary to formalize $S(a, b)$ through an optimization program that minimizes an "error function" that measures how much the values of the inferred parameters contradict the stated holistic preferences. However, $S(a, b)$ is rather "optimization unfriendly". Difficulties arise mainly from the way the non-discordance condition is implemented, i.e., the way $\mathrm{ND}(a, b)$ is defined.

More precisely, two features of the non-discordance relation are concerned. First, the subset of criteria $\bar{F}$ (see (4)) is difficult to integrate into an optimization program. Second, the fact that $C(a, b)$ intervenes in the definition of $\operatorname{ND}(a, b)$ implies that the optimization program will necessarily be non-linear, even when all the parameters are fixed except the weights.

Previous research [3] studying $S(a, b)$ under imprecise information on the criteria weights and veto thresholds has shown that this continuous, non-differentiable, non-linear function is quasi-concave in the domain where it is strictly positive, when $a$ and $b$ are fixed. One consequence of this result is that a constraint like $S(a, b)<\lambda$ (which reflects a holistic statement of the form $\urcorner a S b$ ) does not define a convex set and therefore leads to computationally difficult inference programs.

\section{New ELECTRE-like valued outranking relations}

The definition of the outranking relations presented in this section originated from previous unpublished work that has been presented in [16]. The modified outranking relations $S^{\prime}(a, b)$ and $S^{\prime \prime}(a, b)$ are designed to provide an easier way for $S^{\prime}(a, b)$ and $S^{\prime \prime}(a, b)$ to be integrated in inference optimization programs. Hence, the modifications proposed aim at

1. defining $S^{\prime}(a, b)$ (and $\left.S^{\prime \prime}(a, b)\right)$ as linear functions of the weights $w_{j}$ when the performances $\left(g_{j}(a)\right.$ and $\left.g_{j}(b)\right)$ and thresholds $\left(q_{j}, p_{j}\right.$ and $\left.v_{j}\right)$ are fixed,

2. making $S^{\prime}$ (and $S^{\prime \prime}$ ) as "close" as possible to $S$ both in terms of the results and in terms of the underlying philosophy. 


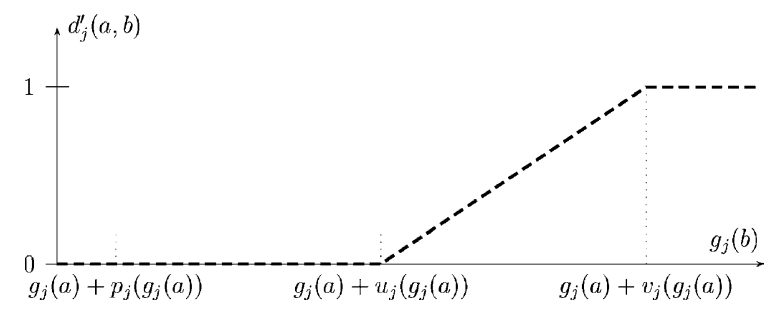

Fig. 3. Partial discordance relation $d_{j}^{\prime}(a, b)$.

More precisely, $S^{\prime}(a, b)$ and $S^{\prime \prime}(a, b)$ will differ from $S(a, b)$ only by its implementation of the discordance concept: new non-discordance relations are defined $\mathrm{ND}^{\prime}(a, b)$ and $\mathrm{ND}^{\prime \prime}(a, b)$ (the outranking relations restricted to a single criterion $S_{j}(a, b)$ and the overall concordance relation $C(a, b)$ remain identical). Furthermore, we will define $S^{\prime}(a, b)=C(a, b) \mathrm{ND}^{\prime}(a, b)$ and $S^{\prime \prime}(a, b)=C(a, b) \mathrm{ND}^{\prime \prime}(a, b)$. Moreover, it should be noted that the way $S^{\prime}\left(S^{\prime \prime}\right.$, respectively) is defined implies $S=S^{\prime}\left(S=S^{\prime \prime}\right.$, respectively) when veto phenomena are either totally effective or totally ineffective (i.e., $\forall a, b$ such that $g_{j}(b)-g_{j}(a) \notin\left[p_{j}, v_{j}\right], \forall j$ ).

\subsection{Definitions}

\subsubsection{Partial discordance indices $d_{j}^{\prime}(a, b)$}

The indices $d_{j}^{\prime}(a, b)$ are defined by (9) on the basis of $g_{j}(a), g_{j}(b)$, a veto threshold function $v_{j}\left(g_{j}\right)$ and an additional threshold function $u_{j}\left(g_{j}\right)$ which we call discordance threshold (such that $\left.p_{j}\left(q_{j}\right) \leqslant u_{j}\left(g_{j}\right)<v_{j}\left(g_{j}\right)\right) .{ }^{4}$ $u_{j}\left(g_{j}(a)\right)$ represents the difference of evaluation $g_{j}(b)-g_{j}(a)$ above which the discordance condition starts to weaken concordance $C(a, b)$ in the definition of $S(a, b)$. Hence, $d_{j}^{\prime}(a, b)$ represents the degree to which criterion $g_{j}$ opposes a veto to the assertion $a S b$ (see Fig. 3). This discordance threshold $u_{j}\left(g_{j}\right)$ can be considered either:

- as an additional preferential parameter to be elicited either directly through an interaction with the DM (the DM should answer questions of the following type: "consider two alternatives $a$ and $b$ such that $a$ is at least as good as $b$ for a majority of criteria and such that $g_{j}(a)<g_{j}(b)$. What should be the minimum difference $g_{j}(b)-g_{j}(a)$ for which a veto phenomenon starts to occur?'), or indirectly using a disaggregation procedure, or

- as a fixed technical parameter (rather than a preference-related one) that defines the extent to which differences of evaluation $g_{j}(b)-g_{j}(a)<v_{j}\left(g_{j}(a)\right)$ should (or should not) weaken the concordance $C(a, b)$ in the definition of $S(a, b)$ (a reasonable value for $u_{j}$ depending on $p_{j}$ and $v_{j}$ is discussed in Section 3.2).

$$
d_{j}^{\prime}(a, b)=1-\frac{v_{j}\left(g_{j}(a)\right)-\min \left\{g_{j}(b)-g_{j}(a), v_{j}\left(g_{j}(a)\right)\right\}}{v_{j}\left(g_{j}(a)\right)-\min \left\{g_{j}(b)-g_{j}(a), u_{j}\left(g_{j}(a)\right)\right\}} .
$$

\subsubsection{Overall non-discordance relation $N D^{\prime}(a, b)$}

The valued non-discordance relation $\operatorname{ND}^{\prime}(a, b)$ is grounded on the relations $d_{j}^{\prime}(a, b), j=1,2, \ldots, n$. It is defined hereafter in (10). It should be noted that criteria that intervene in the product are not restricted to those for which $d_{j}^{\prime}(a, b)>C(a, b)$, i.e., small values of $d_{j}^{\prime}(a, b)$ will impact $\mathrm{ND}^{\prime}(a, b)$. Moreover, the concordance relation $C(a, b)$ does not intervene in the non-discordance implementation.

\footnotetext{
${ }^{4}$ We consider $u_{j}\left(g_{j}\right)<v_{j}\left(g_{j}\right)$ although $u_{j}\left(g_{j}\right)=v_{j}\left(g_{j}\right)$ can also be considered as an extreme case in which discordance is effective only when the veto threshold is exceeded.
} 


$$
\mathrm{ND}^{\prime}(a, b)=\prod_{j \in F}\left(1-d_{j}^{\prime}(a, b)\right) .
$$

In order to study how $\mathrm{ND}^{\prime}(a, b)$ compares to $\mathrm{ND}(a, b)$, let us pose the following lemma.

\section{Lemma. It holds}

$$
1-d_{j}^{\prime}(a, b)=\min \left\{1, \frac{1-d_{j}(a, b)}{1-\alpha_{j}}\right\}
$$

where $\alpha_{j}$ is such that $u_{j}=p_{j}+\alpha_{j} \cdot\left(v_{j}-p_{j}\right)$.

Proof. Let us first define the function $U(x)=\max \{0, \min \{1, x\}\}$. It is easy to verify that: (i) $1-U(x)=U(1-x)$, and (ii) $\forall \varphi \geqslant 0, U(\varphi x)=\min \{1, \varphi U(x)\}$.

To prove this lemma, note first that

$$
d_{j}(a, b)=U\left(\frac{g_{j}(b)-g_{j}(a)-p_{j}\left(g_{j}(a)\right)}{v_{j}\left(g_{j}(a)\right)-p_{j}\left(g_{j}(a)\right)}\right)
$$

(see (3) and Fig. 2) whereas

$$
d_{j}^{\prime}(a, b)=U\left(\frac{g_{j}(b)-g_{j}(a)-u_{j}\left(g_{j}(a)\right)}{v_{j}\left(g_{j}(a)\right)-u_{j}\left(g_{j}(a)\right)}\right)
$$

(see (9) and Fig. 3).

From (i),

$$
1-d_{j}(a, b)=U\left(1-\frac{g_{j}(b)-g_{j}(a)-p_{j}\left(g_{j}(a)\right)}{v_{j}\left(g_{j}(a)\right)-p_{j}\left(g_{j}(a)\right)}\right)=U\left(\frac{v_{j}\left(g_{j}(a)\right)-g_{j}(b)+g_{j}(a)}{v_{j}\left(g_{j}(a)\right)-p_{j}\left(g_{j}(a)\right)}\right) .
$$

On the other hand,

$$
\begin{aligned}
1-d_{j}^{\prime}(a, b) & =U\left(1-\frac{g_{j}(b)-g_{j}(a)-u_{j}\left(g_{j}(a)\right)}{v_{j}\left(g_{j}(a)\right)-u_{j}\left(g_{j}(a)\right)}\right)=U\left(\frac{v_{j}\left(g_{j}(a)\right)-g_{j}(b)+g_{j}(a)}{v_{j}\left(g_{j}(a)\right)-u_{j}\left(g_{j}(a)\right)}\right) \\
& =U\left(\frac{v_{j}\left(g_{j}(a)\right)-p_{j}\left(g_{j}(a)\right)}{v_{j}\left(g_{j}(a)\right)-u_{j}\left(g_{j}(a)\right)} \frac{v_{j}\left(g_{j}(a)\right)-g_{j}(b)+g_{j}(a)}{v_{j}\left(g_{j}(a)\right)-p_{j}\left(g_{j}(a)\right)}\right)
\end{aligned}
$$

where

$$
\frac{v_{j}\left(g_{j}(a)\right)-p_{j}\left(g_{j}(a)\right)}{v_{j}\left(g_{j}(a)\right)-u_{j}\left(g_{j}(a)\right)}=\frac{1}{1-\alpha_{j}}>0 .
$$

Using (ii), it holds

$$
1-d_{j}^{\prime}(a, b)=\min \left\{1, \frac{1}{1-\alpha_{j}} U\left(\frac{v_{j}\left(g_{j}(a)\right)-g_{j}(b)+g_{j}(a)}{v_{j}\left(g_{j}(a)\right)-p_{j}\left(g_{j}(a)\right)}\right)\right\}=\min \left\{1, \frac{1}{1-\alpha_{j}}\left(1-d_{j}(a, b)\right)\right\} .
$$

According to the preceding lemma, the valued non-discordance relation $\operatorname{ND}^{\prime}(a, b)$ can be defined equivalently as formulated in (12) and (13), where $\alpha_{j} \in[0,1], j \in F$ is a parameter that should be defined such that $u_{j}=p_{j}+\alpha_{j} \cdot\left(v_{j}-p_{j}\right)$. 


$$
\begin{aligned}
& \mathrm{ND}^{\prime}(a, b)=\prod_{j \in F} \mathrm{ND}_{j}^{\prime}(a, b), \\
& \mathrm{ND}_{j}^{\prime}(a, b)=\min \left\{1, \frac{1-d_{j}(a, b)}{1-\alpha_{j}}\right\} .
\end{aligned}
$$

This alternative definition of $\operatorname{ND}_{j}^{\prime}(a, b)$ when compared to (6), shows that the parameters $\alpha_{j}$ plays the same role in (13) that the term $C(a, b)$ in the denominator of (6). In other words, the modification introduced in $S^{\prime}(a, b)$ as compared to $S(a, b)$ amounts at replacing $C(a, b)$ in (6) by a value $\alpha_{j}$ that defines the "position" of the threshold $u_{j}\left(g_{j}\right)$ in the interval $\left[p_{j}\left(g_{j}\right), v_{j}\left(g_{j}\right)\right]\left(u_{j}=p_{j}+\alpha_{j} \cdot\left(v_{j}-p_{j}\right)\right)$. Note that the value of $\alpha_{j}$ is the same for every pair $(a, b) \in A^{2}$ whereas $C(a, b)$ varies. The DM may either fix $u_{j}$ directly, or indirectly by fixing $\alpha_{j}$.

\subsubsection{Overall non-discordance relation $N D^{\prime \prime}(a, b)$}

The valued non-discordance relation $\operatorname{ND}^{\prime \prime}(a, b)$ is grounded on the relations $d_{j}^{\prime}(a, b), j=1,2, \ldots, n$. It is defined hereafter in (14). It should be noted that criteria that intervene in the Min operator are not restricted to those for which $d_{j}^{\prime}(a, b)>C(a, b)$. Moreover, the concordance relation $C(a, b)$ does not intervene in the non-discordance implementation.

$$
\mathrm{ND}^{\prime \prime}(a, b)=\operatorname{Min}_{j \in F}\left(1-d_{j}^{\prime}(a, b)\right) \text {. }
$$

\subsection{Comparative analysis}

As already mentioned, $S(a, b), S^{\prime}(a, b)$ and $S^{\prime \prime}(a, b)$ differ only on the way the non-discordance condition is implemented. Let us recall that the modifications intervening in $S^{\prime}(a, b)$ and $S^{\prime \prime}(a, b)$ aim at providing easier ways to integrate the outranking relation in disaggregation procedures. It should be emphasized that $S^{\prime}(a, b)$ is closely related to $S(a, b)$; the similarity appears when comparing (13) and (6). As $S^{\prime \prime}(a, b)$ is very similar to $S^{\prime}(a, b)\left(S^{\prime \prime}(a, b)\right.$ account for the "strongest" veto, whereas $S^{\prime}(a, b)$ can account for several veto effects, as $S(a, b)$ does), we will mainly focus on the comparison between $S(a, b)$ and $S^{\prime}(a, b)$. Two modifications have been introduced in $S^{\prime}$ as compared to $S$.

- The relation $\operatorname{ND}^{\prime}(a, b)$ does not account for $C(a, b)$. Hence a veto situation is not accentuated when the concordance relation is not firmly established as it is the case in $\operatorname{ND}(a, b)$. Although disabling this feature removes some refinements, it also distinguishes more clearly the way the two concepts of concordance and non-discordance are implemented. Moreover, a low value for $C(a, b)$ still impacts directly $S^{\prime}(a, b)$, but the value of $C(a, b)$ does not impact $\mathrm{ND}^{\prime}(a, b)$ (note that $C(a, b)$ does impact $\mathrm{ND}(a, b)$ ).

- As in $S(a, b), S^{\prime}(a, b)$ avoids to account for discordance situations that are not firmly established. Such feature is done in $S(a, b)$ by accounting in $\operatorname{ND}(a, b)$ for discordant criteria such that $d_{j}(a, b)>C(a, b)$ only. $S^{\prime}(a, b)$ also does not account for "weak veto": although $\mathrm{ND}^{\prime}(a, b)$ considers all discordant criteria $\left(\mathrm{ND}^{\prime}(a, b)=\prod_{j \in F} 1-d_{j}^{\prime}(a, b)\right)$, the way $d_{j}^{\prime}(a, b)$ are defined induce lower values (as compared to $d_{j}(a, b)$ ) for the same difference $g_{j}(b)-g_{j}(a)$, namely $d_{j}^{\prime}(a, b)=0 \forall a, b \in A$, such that $g_{j}(b)-g_{j}(a) \leqslant u_{j}\left(g_{j}(a)\right)$. Figs. 4 and $5^{5}$ show how $\operatorname{ND}(a, b)$ and $\operatorname{ND}^{\prime}(a, b)$ vary as a function of $g_{j}(b)$ when $g_{j}$ is the only discordant criterion, i.e., depict $\mathrm{ND}_{j}(a, b)$ and $\mathrm{ND}_{j}^{\prime}(a, b)$.

\footnotetext{
${ }^{5}$ In Fig. 5, $x$ corresponds to $p_{j}\left(g_{j}(a)\right)+C(a, b) \cdot\left(v_{j}\left(g_{j}(a)\right)-p_{j}\left(g_{j}(a)\right)\right)$ and hence varies in the interval $\left[p_{j}\left(g_{j}(a)\right), v_{j}\left(g_{j}(a)\right)\right]$ as $C(a, b)$ varies in $[0,1]$.
} 


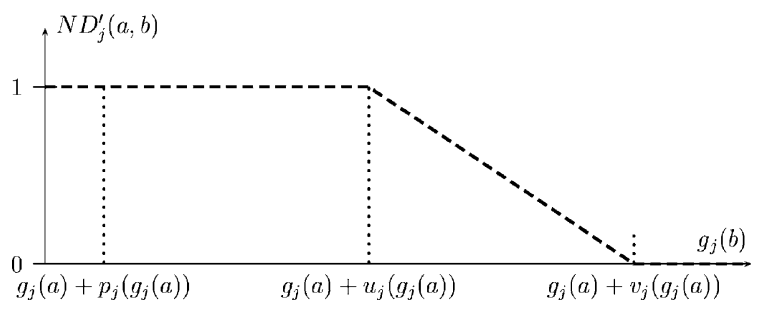

Fig. 4. $\mathrm{ND}_{j}^{\prime}(a, b)$ as a function of $g_{j}(b)$.

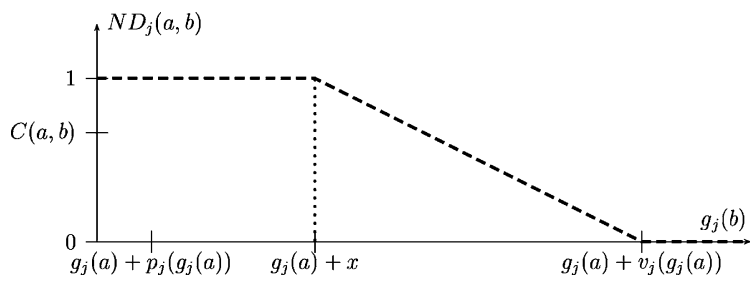

Fig. 5. $\mathrm{ND}_{j}^{\prime}(a, b)$ as a function of $g_{j}(b)$.

One important question related to the way $S(a, b)$ and $S^{\prime}(a, b)$ implement discordance is the following: if $u_{j}$ is not considered as a preference parameter, how should it be defined in order for $S(a, b)$ and $S^{\prime}(a, b)$ to be "as close as possible"?

Let us remark that, for $S(a, b)$ and $S^{\prime}(a, b)$, it holds

- discordance operates by weakening $C(a, b)$,

- for any $\lambda$-cut $S_{\lambda}\left(S_{\lambda}^{\prime}\right.$, respectively) of $S(a, b)\left(S^{\prime}(a, b)\right.$, respectively), the assertion $a S_{\lambda} b$ ( $a S_{\lambda}^{\prime} b$, respectively) cannot hold for any $(a, b)$ such that $C(a, b)<0.5$.

It follows from these two preliminary remarks that it is sufficient so as to compare $S(a, b)$ and $S^{\prime}(a, b)$ to restrict the analysis to the pairs $(a, b)$ such that $C(a, b) \geqslant 0.5$.

In order for $S^{\prime}(a, b)$ to be "close" to $S(a, b), \mathrm{ND}(a, b)$ should not differ too much from $\mathrm{ND}^{\prime}(a, b)$, i.e., each $\mathrm{ND}_{j}(a, b)$ should not be far from $\mathrm{ND}_{j}^{\prime}(a, b)$. Hence, we should define $u_{j}$ so that $f\left(C(a, b), g_{j}(b)\right)=\left|\mathrm{ND}_{j}(a, b)-\mathrm{ND}_{j}^{\prime}(a, b)\right|$ is as small as possible in average, when $C(a, b) \in[0.5,1]$ and $g_{j}(b) \in\left[g_{j}(a)+p_{j}\left(g_{j}(a)\right), g_{j}(a)+v_{j}\left(g_{j}(a)\right)\right]$ (Figs. 6 and 7 represent ND ND $_{j}$ and $\mathrm{ND}_{j}^{\prime}$ as functions of $C(a, b)$ and $\left.g_{j}(b)\right)$. In order to do so we should set $\alpha_{j}=0.75$, i.e., $u_{j}=p_{j}\left(g_{j}(a)\right)+0.75 .\left(v_{j}\left(g_{j}(a)\right)-p_{j}\left(g_{j}(a)\right)\right)$. The value $\alpha_{j}=0.75$ is obtained by minimizing the following expression:

$$
\operatorname{Min}_{\alpha \in[0,1]}\left\{\int_{0.5}^{1} \int_{g_{j}(a)+p_{j}\left(g_{j}(a)\right)}^{g_{j}(a)+v_{j}\left(g_{j}(a)\right)} f\left(C(a, b), g_{j}(b), \alpha\right) d C(a, b) d g_{j}(b)\right\} .
$$

Alternatively, it is possible to define $\alpha_{j}$ as equal to the cutting level $\lambda$ (when the value for $\lambda$ is fixed and known). This ensures that any $\lambda$-cut of $S$ and $S^{\prime}$ are identical, but requires to determine the value for $\lambda$ beforehand.

In order to appreciate the effective deviation between $S(a, b), S^{\prime}(a, b)$ and $S^{\prime \prime}(a, b)$, we have performed an empirical study on datasets stemming from real world case studies reported in the literature [6,28] $(139$ alternatives in total; these tests were performed fixing $\alpha_{j}=0.75 \forall j \in F$ ). More specifically, we observe how much $S^{\prime}(a, b)$ and $S^{\prime \prime}(a, b)$ deviate from $S(a, b)$ on real data and analyze to what extent substituting $S^{\prime}(a, b)$ 


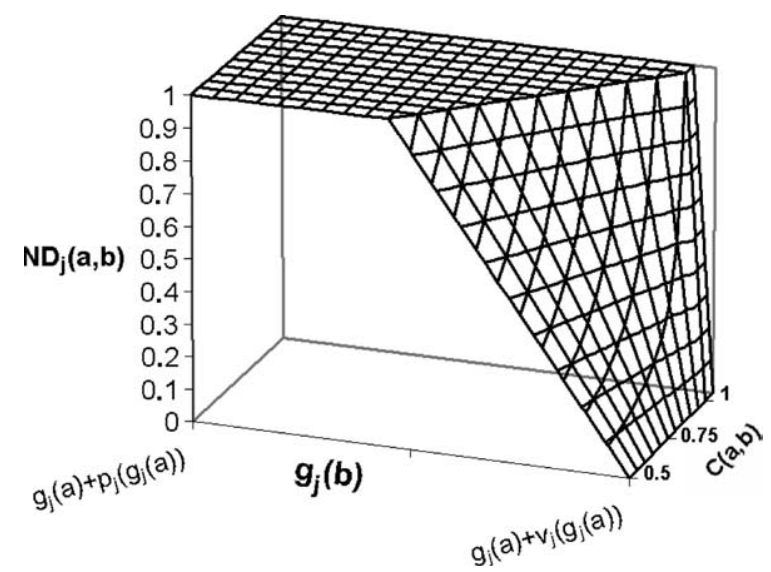

Fig. 6. $\mathrm{ND}_{j}(a, b)$ as a function of $g_{j}(b)$ and $C(a, b)$.

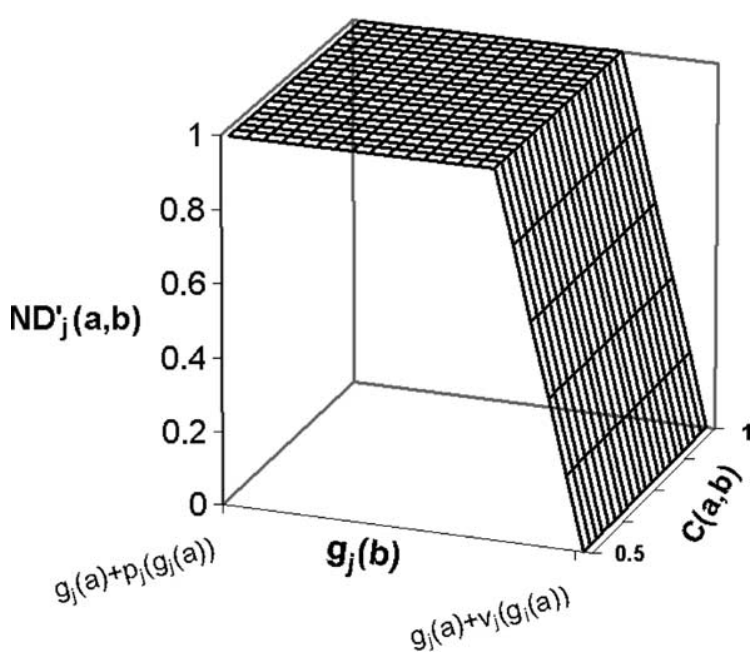

Fig. 7. $\mathrm{ND}_{j}^{\prime}(a, b)$ as a function of $g_{j}(b)$ and $C(a, b)$.

(or $S^{\prime \prime}(a, b)$ ) for $S(a, b)$ affects the conclusions of these studies, which concerned assigning alternatives to categories using ELECTRE TRI. Considering [6,28], we verified that the assignments of all alternatives are identical using $S, S^{\prime}$ and $S^{\prime \prime}$. The maximum deviation (i.e., $\left|S(a, b)-S^{\prime}(a, b)\right|$ and $\left|S(a, b)-S^{\prime \prime}(a, b)\right|$, $a \in A, b \in B)$ is equal to 0.1 . Although it is possible to design specific situations in which the assignments using $S^{\prime}(a, b)$ (or $S^{\prime \prime}(a, b)$ ) and $S(a, b)$ are different, our experiments show that such cases do not occur frequently on the data considered $[6,28]$.

\section{Benefit of the revised index with respect to parameter inference programs}

Assigning values to the parameters involved in the definition of $S^{\prime}$ and $S^{\prime \prime}$ is a difficult task for the DM. The disaggregation approach (see [8]) allows to infer preferential parameter values from holistic prefer- 
ences. Such approach is usually performed using mathematical programs that minimize an "error function". By inference programs, we mean mathematical programs aiming at determining values for preference parameters involved in $S(a, b)$ from holistic preferences provided by the DM. Such inference programs can either be partial if only a subset of parameters is being inferred (the values of the other parameters being fixed), or global if all parameters are to be inferred. In this section, we will illustrate the reduction in the complexity of the mathematical programs to be solved when substituting $S^{\prime}$ or $S^{\prime \prime}$ for $S$ on what concerns the inference of the weights and the cutting level.

\subsection{Inferring a valued outranking relation from crisp outranking statements}

\subsubsection{Global inference program}

In this section we will consider a decision process in which an outranking relation is used to model DMs preferences. Furthermore, let us suppose that the DM is not able (or not willing) to assign directly values to the preference-related parameters involved in the outranking relation, but can state crisp statements about this relation for some specific pairs of alternatives $(a, b)$, i.e., either $a S b$ or $\urcorner a S b$. Our purpose is to define a valued outranking relation and a cutting level that best account for the DM statements.

Let us denote $S^{+}=\left\{(a, b) \in A^{2}\right.$ such that the DM stated $\left.a S b\right\}$ and $S^{-}\left\{(a, b) \in A^{2}\right.$ such that the DM stated $\neg a S b$. Then, a combination of parameter values is able to restore the DM's request iff $S(a, b) \geqslant \lambda$ $\forall(a, b) \in S^{+}$and $S(a, b)<\lambda \forall(a, b) \in S^{-}$, which may be written as $S(a, b)-\lambda \geqslant 0 \forall(a, b) \in S^{+}$and $\lambda-S(a, b)+\varepsilon \geqslant 0 \forall(a, b) \in S^{-}$( $\varepsilon$ being a small positive value). The mathematical program given below (15)-(20) maximizes a common slack $\alpha$ for all these constraints, to obtain a relatively "central" combination of parameter values. Whenever the optimum value of $\alpha$ is negative, there is no combination of parameter values complying to all the constraints, i.e., the DM provided inconsistent information (a procedure to deal with such inconsistencies is proposed in [14]). Alternative objective functions can be considered (see $[1,17])$ :

$$
\begin{array}{ll}
\text { Max } & \alpha \\
\text { s.t. } & \alpha \leqslant S(a, b)-\lambda \quad \forall(a, b) \in S^{+}, \\
& \alpha \leqslant \lambda-S(a, b)+\varepsilon \quad \forall(a, b) \in S^{-}, \\
& \lambda \in[0.5,1], \\
& v_{j}\left(g_{j}\right)>p_{j}\left(g_{j}\right)>q_{j}\left(g_{j}\right) \geqslant 0 \quad \forall j \in F, \\
& \sum_{j=1}^{n} w_{j}=1 ; \quad w_{j} \geqslant \varepsilon \quad \forall j \in F .
\end{array}
$$

Some additional constraints can be added to this program, in order to integrate explicit statements of the DM concerning the values of some parameters. From (4) and (8), it is obvious that this is a difficult nonlinear program when all the parameters are considered as variables (recall Section 2.6.2). A solution to circumvent this difficulty is to formulate partial inference programs, where only a subset of the parameters are considered as variables, while the remaining ones are fixed. In the context of a decision aiding process where the DM interactively revise the information they provide and observe the results of the mathematical program, partial inference problems allow them to focus their attention on a subset of parameters at a time and to better understand the consequences of their modifications. Indeed, we believe that inference programs should not be considered as a problem to be solved once, but rather as problems to be solved many times in an interactive learning process. Among the partial inference problems, previous research on related problems has focused mainly on inferring the weights and the cutting level (see $[5,13,18])$. This is an important partial inference problem because the weights and the cutting level are the only parameters involving inter-criteria judgements (the remaining parameters do not interrelate the criteria). 


\subsubsection{Inferring the weights $\left(w_{j}, j \in F\right)$ and cutting level $(\lambda)$ only}

If we consider the case where only the weights $\left(w_{j}, j \in F\right)$ and cutting level $(\lambda)$ are variables (all other parameters being fixed), then the constraints (16) and (17) can be rewritten in as in (21) and (22). These two constraints are obviously nonlinear, since they represent products of functions involving $C(a, b)$, which in turn involve the $w_{j}$ variables (see (2)).

$$
\begin{aligned}
& \alpha \leqslant C(a, b) \prod_{j \in F} \operatorname{Min}\left\{1, \frac{1-d_{j}(a, b)}{1-C(a, b)}\right\}-\lambda \quad \forall(a, b) \in S^{+}, \\
& \alpha \leqslant \lambda-C(a, b) \prod_{j \in F} \operatorname{Min}\left\{1, \frac{1-d_{j}(a, b)}{1-C(a, b)}\right\}+\varepsilon \quad \forall(a, b) \in S^{-} .
\end{aligned}
$$

Let us now consider the same problem when $S(a, b)$ is substituted by $S^{\prime}(a, b)$. In this case, the constraints (16) and (17) become (23) and (24). Now, each $\prod_{j \in F}\left(1-d_{j}^{\prime}(a, b)\right)=\mathrm{ND}^{\prime}(a, b)$ is a fixed constant $\forall(a, b)$. The constraints (23) and (24) are hence linear, since $C(a, b)$ is an affine function of the weights.

$$
\begin{aligned}
& \alpha \leqslant C(a, b) \prod_{j \in F}\left(1-d_{j}^{\prime}(a, b)\right)-\lambda \quad \forall(a, b) \in S^{+}, \\
& \alpha \leqslant \lambda-C(a, b) \prod_{j \in F}\left(1-d_{j}^{\prime}(a, b)\right)+\varepsilon \quad \forall(a, b) \in S^{-} .
\end{aligned}
$$

Thus, considering $S^{\prime}(a, b)$ instead of $S(a, b)$, the weights and the cutting level can be inferred by solving a linear program whose variables are $\alpha, w_{1}, \ldots, w_{n}$, and $\lambda$, where (23) and (24) appear as (26) and (27):

$$
\begin{array}{ll}
\text { Max } & \alpha \\
\text { s.t. } & \alpha \leqslant \sum_{j=1}^{n} w_{j} S_{j}(a, b) \mathrm{ND}^{\prime}(a, b)-\lambda \quad \forall(a, b) \in S^{+}, \\
& \alpha \leqslant \lambda-\sum_{j=1}^{n} w_{j} S_{j}(a, b) \operatorname{ND}^{\prime}(a, b)+\varepsilon \quad \forall(a, b) \in S^{-}, \\
& \lambda \in[0.5,1], \\
& \sum_{j=1}^{n} w_{j}=1 \quad w_{j} \geqslant \varepsilon \quad \forall j \in F .
\end{array}
$$

If the maximum value of $\alpha$ is positive, then the values of $w_{1}, \ldots, w_{n}$, and $\lambda$ at the optimum are able to restore all the statements defining $S^{+}$and $S^{-}$. Otherwise, the inferred values provide suggestions for changing those examples. The DM should ponder whether they want to change the sets $S^{+}$and $S^{-}$, or to analyze the values of $\mathrm{ND}^{\prime}(a, b)$. Indeed, some of the differences among the current model and the DM's requests may stem from inadequate values for the veto and discordance thresholds. Considering $S^{\prime \prime}(a, b)$ instead of $S^{\prime}(a, b)$ leads to a similar linear program.

To infer relevant values for $w_{j}$ and $\lambda$, the cardinality of $S^{+}$and $S^{-}$should be "sufficiently" large; [15] presents an experimental study providing useful information about this point. Multiple optima can occur in these mathematical programs. However, inference procedures are to be used iteratively in disaggregation processes. During this interaction, the DM can change some of this input and learn about his/her preferences while exploring the different solutions. 


\subsection{Inferring ELECTRE TRI parameters from assignment examples}

\subsubsection{Brief reminder on ELECTRE TRI}

ELECTRE TRI (see $[25,28]$ ) assigns alternatives to pre-defined ordered categories. The assignment of an alternative $a \in A$ results from comparing it with the profiles defining the limits of the categories. Let $B=\left\{b_{1}, b_{2}, \ldots, b_{p}\right\}$ denote the set of profiles defining $p+1$ categories, $b_{h}$ being the upper limit of category $C_{h}$ and the lower limit of category $C_{h+1}, h=1,2, \ldots, p$ (profiles $b_{p+1}$ and $b_{0}$ correspond to the ideal and antiideal alternatives, respectively). Let $K=\left\{C_{1}, C_{2}, \ldots, C_{p+1}\right\}$ be the set of categories that corresponds to $B$. ELECTRE TRI assigns alternatives to categories following two consecutive steps:

- construction of a crisp outranking relation $S \subset(A \times B) \cup(B \times A)$; the definition of $S$ corresponds to $\lambda$ cut of $S(a, b)$ described in Section 2, except that the thresholds $q_{j}, p_{j}$ and $v_{j}$, are values attached to the profiles $\left(q_{j}\left(b_{h}\right), p_{j}\left(b_{h}\right)\right.$ and $\left.v_{j}\left(b_{h}\right) \forall j \in F \forall b_{h} \in B\right)$, rather than dependent on the alternatives in $A$.

- exploitation of the crisp relation $S$ in order to assign each alternative to a specific category using a pessimistic or optimistic procedure (in what follows we will restrict our analysis to the pessimistic procedure). The pessimistic procedure is defined as follows:

(a) compute $S\left(a, b_{i}\right)$ successively for $i=p, p-1, \ldots, 0$,

(b) $b_{h}$ being the first profile such that $S\left(a, b_{h}\right) \geqslant \lambda$, assign $a$ to category $C_{h+1}\left(a \rightarrow C_{h+1}\right)$.

Hence, the pessimistic procedure of ELECTRE TRI assigns alternative $a$ to category $C_{h}\left(b_{h-1}\right.$ and $b_{h}$ being the lower and upper profiles of $C_{h}$, respectively) iff $S\left(a, b_{h-1}\right) \geqslant \lambda$ and $S\left(a, b_{h}\right)<\lambda(\lambda \in[0.5,1]$ is the chosen cutting level).

\subsubsection{Inference programs}

Suppose the DM has specified a set of assignment examples, i.e., a subset of $A^{*} \subset A$ such that each $a_{k} \in A^{*}$ is associated with $C^{M}\left(a_{k}\right)\left(C^{m}\left(a_{k}\right)\right.$, respectively) the maximum (minimum, respectively) category to which $a$ should be assigned according to his/her holistic preferences. Hence $\left[C^{m}\left(a_{k}\right), C^{M}\left(a_{k}\right)\right]$ defines an interval of possible categories to which $a_{k}$ can be assigned to. $C^{m}\left(a_{k}\right)=C^{M}\left(a_{k}\right)=C_{h_{k}}$ means that the DM wants $a_{k}$ to be assigned to $C_{h_{k}}$ precisely (we will note $a_{k} \rightarrow_{D M} C_{h_{k}}$ such statement), while $C^{m}\left(a_{k}\right)<C^{M}\left(a_{k}\right)$ corresponds to an imprecise statement $\left(a_{k} \rightarrow_{D M}\left[C^{m}\left(a_{k}\right), C^{M}\left(a_{k}\right)\right]\right)$. Inferring all ELECTRE TRI parameters can be formulated through the following mathematical program (30)-(36), see [17]. Note that all alternatives in $A^{*}$ are assigned by ELECTRE TRI (using the inferred parameters) consistently with the DM examples if and only if the optimal value of the objective function is positive.

$$
\begin{array}{ll}
\text { Max } & \alpha \\
\text { s.t. } & \alpha \leqslant S\left(a_{k}, b_{C^{m}\left(a_{k}\right)-1}\right)-\lambda \quad \forall a_{k} \in A^{*}, \\
& \alpha \leqslant \lambda-S\left(a_{k}, b_{C^{M}\left(a_{k}\right)}\right)+\varepsilon \quad \forall a_{k} \in A^{*}, \\
& \lambda \in[0.5,1], \\
& g_{j}\left(b_{h+1}\right) \geqslant g_{j}\left(b_{h}\right)+p_{j}\left(b_{h}\right)+p_{j}\left(b_{h+1}\right) \quad \forall j \in F \quad \forall b_{h} \in B, \\
& v_{j}\left(b_{h}\right) \geqslant p_{j}\left(b_{h}\right) \geqslant q_{j}\left(b_{h}\right) \geqslant 0 \quad \forall j \in F \quad \forall b_{h} \in B, \\
& \sum_{j=1}^{n} w_{j}=1, \quad w_{j} \geqslant \varepsilon, \quad \forall j \in F .
\end{array}
$$

The constraint (34) is introduced to ensure the consistency of category definition, see [18,28]. If we consider all preference parameters as variables, this mathematical program is nonlinear due to constraints (31) and (32). Indeed, the constraints (31) and (32) are similar to the constraints (16) and (17) in the mathematical program presented in Section 4.1.1. 
If we consider the case where only the weights $\left(w_{j}, j \in F\right)$ and cutting level $(\lambda)$ are variables (all other parameters being fixed), then the constraints (31) and (32) will be similar to (21) and (22), hence the mathematical program (30)-(36) remains nonlinear. Previous work [5,18] presented linear programming formulations assuming that there was no discordance, i.e. $S(a, b)=C(a, b)$. One of the motivations for considering $S^{\prime}(a, b)$ instead of $S(a, b)$ is that it becomes easy to infer the weights and the cutting level even when the veto-related parameters make $S(a, b)<C(a, b)$.

Indeed, when considering $S^{\prime}(a, b)$, the weights and the cutting level can be inferred by solving a linear program whose variables are $\alpha, w_{1}, \ldots, w_{n}$, and $\lambda$. The linear program for this partial inference problem is equal to (25)-(29) in Section 4.1.2, if we define

$$
\begin{aligned}
& S^{+}=\left\{\left(a_{k}, b_{C^{m}\left(a_{k}\right)-1}\right) \in A^{*} \times B: a_{k} \rightarrow_{D M}\left[C^{m}\left(a_{k}\right), C^{M}\left(a_{k}\right)\right]\right\}, \\
& S^{-}=\left\{\left(a_{k}, b_{C^{m}\left(a_{k}\right)}\right) \in A^{*} \times B: a_{k} \rightarrow_{D M}\left[C^{m}\left(a_{k}\right), C^{M}\left(a_{k}\right)\right]\right\} .
\end{aligned}
$$

Considering $S^{\prime \prime}(a, b)$ instead of $S^{\prime}(a, b)$ leads to a similar linear program.

\subsection{Miettinen and Salminen ELECTRE like method [13]}

Miettinen and Salminen [13] proposed a method using the ELECTRE III valued outranking relation that aims at "providing the DM descriptive information about the weighting vectors producing a specific alternative as the best", i.e., placed first in a preference ranking. Such procedure requires to solve inference programs. In [13], the preference rankings do not result from the same exploitation procedure as in ELECTRE III (see [23]), but from the use of the "min" procedure (see [22]), i.e., alternatives are ranked based on the minimum outranking degree $S(a, b)$ of each alternative $a \forall b \in A$. In this approach, checking whether or not an alternative $a \in A$ can be ranked first for at least a weight vector amounts at verifying whether the constraints (39) and (40) define a consistent system. Such analysis is performed with fixed values for all the parameters except the weights $w_{j}$. Furthermore, the problem is rather difficult because the constraints (39) and (40) are nonlinear, unless the $v_{j}\left(g_{j}\right)$ are fixed sufficiently large so as no veto phenomenon to occur, i.e., unless discordance is removed from the model.

$$
\begin{aligned}
& \operatorname{Min}_{a^{\prime} \in A \backslash\{a\}}\left\{S\left(a, a^{\prime}\right)\right\}-\operatorname{Min}_{a^{\prime} \in A \backslash\{b\}}\left\{S\left(b, a^{\prime}\right)\right\} \geqslant \varepsilon \quad \forall b \in A \backslash\{a\}, \\
& \varepsilon>0, \sum_{j \in F} w_{j}=1, \quad w_{j} \geqslant 0, \quad \forall j \in F .
\end{aligned}
$$

Different objective functions to optimize subject to (39) and (40) are proposed in [13]. Optimization programs are built in order to

- determine ranges for $w_{j}$ such that $a$ is ranked first (Max and Min $w_{j}$, s.t. (39) and (40)),

- rank $a$ first in the most "robust" way (Max $\varepsilon$ s.t. (39) and (40)),

- rank $a$ first without omitting any criterion (Max Min $w_{j}$ s.t. (39) and (40)).

If discordance is removed from the model, then these optimization programs can be solved using standard linear programming techniques. ${ }^{6}$ However, if we replace $S(a, b)$ by $S^{\prime}(a, b)$ in the preceding optimization programs, it becomes obvious that the restriction that [13] impose on discordance $\left(v_{j}\left(g_{j}(a)\right)>g_{j}(b)-g_{j}(a), \forall a, b \in A \forall j \in F\right)$ is no longer necessary to simplify the problem. In fact, considering $S^{\prime}(a, b)$, it is possible to solve these optimization programs using linear programming even when

\footnotetext{
${ }^{6}$ This requires to add supplementary variables to account for the min operator in (39).
} 
veto phenomena occur (the $v_{j}$ functions being defined). The same statement can be made when considering $S^{\prime \prime}(a, b)$ instead of $S^{\prime}(a, b)$.

\section{Conclusion}

This paper presents a slight adaptation of the valued outranking relation used in the ELECTRE III (see [23]) and ELECTRE TRI (see [25,28]); the modifications introduced concern the implementation of the non-discordance condition in the outranking relation. The two new outranking relations $S^{\prime}$ and $S^{\prime \prime}$ preserve the original ideas (namely the original discordance concept) and are designed to be more optimizationfriendly for parameter inference programs. We show that the modified outranking relation makes it easier to solve inference programs. Relations $S^{\prime}$ and $S^{\prime \prime}$ are equivalent as regards the complexity of inferring the weights and cutting level, although $S^{\prime \prime}$ is more friendly in what regards inferring veto thresholds (as we show in [4]).

\section{Acknowledgements}

This work has benefited from the grant no. 500B4 (ICCTI/Ambassade de France au Portugal). The authors are grateful to Bernard Roy for his comments and remarks on earlier versions of this text.

\section{References}

[1] M. Beuthe, G. Scannella, Comparative analysis of UTA multicriteria methods, European Journal of Operational Research 130 (2) (2001) 246-262.

[2] J.P. Brans, B. Mareschal, Ph. Vincke, PROMÉTHÉE: A new family of outranking methods in multicriteria analysis, in: J.P. Brans (Ed.), Operational Research'84, North-Holland, Elsevier Science Publishers, Amsterdam, 1984, pp. 408-421.

[3] L.C. Dias, J.N. Clímaco, On computing ELECTRE's credibility indices under partial information, Journal of Multi-Criteria Decision Analysis 8 (2) (1999) 74-92.

[4] L.C. Dias, V. Mousseau, Inferring ELECTRE's veto-related parameters from outranking examples. Research report no. 5/2002, INESC Coimbra, 2002.

[5] L.C. Dias, V. Mousseau, J. Figueira, J. Clímaco, An aggregation/disaggregation approach to obtain robust conclusions with ELECTRE TRI, European Journal of Operational Research 138 (2) (2002) 332-348.

[6] M. Doumpos, C. Hurson, A multicriteria decision aid method for the assessment of business failure risk, Foundations of Computing and Decision Sciences 20 (2) (1995) 99-112.

[7] J. Fodor, M. Roubens, Fuzzy Preference Modelling and Multicriteria Decision Support, Kluwer Academic Publishers, Dordrecht, 1994.

[8] E. Jacquet-Lagréze, Y. Siskos, Assessing a set of additive utility functions for multicriteria decision-making, The UTA method, European Journal of Operational Research 10 (2) (1982) 151-164.

[9] E. Jacquet-Lagréze, Y. Siskos, Preference disaggregation, 20 years of MCDA experience, European Journal of Operational Research 130 (2) (2001) 233-245.

[10] R. Keeney, H. Raiffa, Decision with Multiple Objectives: Preferences and Value Tradeoffs, John Wiley and Sons, New York, 1976.

[11] L.N. Kiss, J.-M. Martel, R. Nadeau, ELECCALC — an interactive software for modelling the decision maker's preferences, Decision Support Systems 12 (4-5) (1994) 757-777.

[12] J.-P. Leclercq, Propositions d'extension de la notion de dominance en présence de relations d'ordre sur les pseudo-critères: Melchior, Revue Belge de Recherche Opérationnelle de Statistique et d'Informatique 24 (1) (1984) 32-46.

[13] K. Miettinen, P. Salminen, Decision-aid for discrete multiple criteria decision making problems with imprecise data, European Journal of Operational Research 119 (1) (1999) 50-60.

[14] V. Mousseau, L.C. Dias, J. Figueira, C. Gomes, J. Clímaco, Resolving inconsistencies among constraints on the parameters of an MCDA model, European Journal of Operational Research 143 (1) (2003) 332-348.

[15] V. Mousseau, J. Figueira, J.-Ph. Naux, Using assignment examples to infer weights for ELECTRE TRI method: Some experimental results, European Journal of Operational Research 130 (2) (2001) 263-275. 
[16] V. Mousseau, R. Slowinski, L'approche agrégation/désagrégation pour les méthodes de surclassement, Séminaire Modélisation des Préférences et Aide Multicritère à la Décision, LAMSADE, Université de Paris-Dauphine, 1997.

[17] V. Mousseau, R. Slowinski, Inferring an ELECTRE TRI model from assignment examples, Journal of Global Optimization 12 (2) (1998) $157-174$.

[18] V. Mousseau, R. Slowinski, P. Zielniewicz, A user-oriented implementation of the ELECTRE TRI method integrating preference elicitation support, Computers and Operations Research 27 (7-8) (2000) 757-777.

[19] J.H.P. Paelinck, Qualiflex: A flexible multiple-criteria decision method, Economics Letters 1 (1978) $193-197$.

[20] H. Pastijn, J. Leysen, Constructing an outranking relation with ORESTE, Mathematical and Computer Modelling 12 (10-11) (1989) 1255-1268.

[21] P. Perny, B. Roy, The use of fuzzy outranking relations in preference modelling, Fuzzy Sets and Systems 49 (1) (1992) $33-53$.

[22] M. Pirlot, A characterisation of "min" as a procedure for exploiting valued preference relations and related results, Journal of Multi-Criteria Decision Analysis 4 (1995) 37-56.

[23] B. Roy, ELECTRE III: Un algorithme de classement fondé sur une représentation floue des préférences en présence de critères multiples, Cahiers du CERO 20 (1) (1978) 3-24.

[24] B. Roy, The outranking approach and the foundations of ELECTRE methods, Theory and Decision 31 (1) (1991) $49-73$.

[25] B. Roy, D. Bouyssou, Aide multicritère à la décision: Méthodes et cas, Economica, Paris, 1993.

[26] B. Roy, D. Vanderpooten, An overview on the the european school of MCDA: Emergence, basic features and current work, European Journal of Operational Research 99 (1) (1997) 26-27.

[27] J.-C1. Vansnick, On the problem of weight in multiple criteria decision making (the noncompensatory approach), European Journal of Operational Research 24 (1986) 288-294.

[28] W. Yu, Aide multicritère à la décision dans le cadre de la problématique du tri: concepts, méthodes et applications. Ph.D. Thesis, Université Paris-Dauphine, 1992. 